\title{
Effects of small surface tension in Hele-Shaw multifinger dynamics: An analytical and numerical study
}

\author{
E. Pauné, ${ }^{1}$ M. Siegel, ${ }^{2}$ and J. Casademunt ${ }^{1}$ \\ ${ }^{1}$ Departament d'Estructura i Constituents de la Matèria, Universitat de Barcelona, Avinguda Diagonal 647, 08028 Barcelona, Spain \\ ${ }^{2}$ Mathematics Department, New Jersey Institute of Technology, Newark, New Jersey 07102
}

(Received 18 April 2002; published 10 October 2002)

\begin{abstract}
We study the singular effects of vanishingly small surface tension on the dynamics of finger competition in the Saffman-Taylor problem, using the asymptotic techniques described by Tanveer [Philos. Trans. R. Soc. London, Ser. A 343, 155 (1993)] and Siegel and Tanveer [Phys. Rev. Lett. 76, 419 (1996)], as well as direct numerical computation, following the numerical scheme of Hou, Lowengrub, and Shelley [J. Comput. Phys. 114, 312 (1994)]. We demonstrate the dramatic effects of small surface tension on the late time evolution of two-finger configurations with respect to exact (nonsingular) zero-surface-tension solutions. The effect is present even when the relevant zero-surface-tension solution has asymptotic behavior consistent with selection theory. Such singular effects, therefore, cannot be traced back to steady state selection theory, and imply a drastic global change in the structure of phase-space flow. They can be interpreted in the framework of a recently introduced dynamical solvability scenario according to which surface tension unfolds the structurally unstable flow, restoring the hyperbolicity of multifinger fixed points.
\end{abstract}

DOI: 10.1103/PhysRevE.66.046205

PACS number(s): 47.54.+r, 47.20.Ma, 47.20.Ky, 47.20.Hw

\section{INTRODUCTION}

The displacement of a viscous fluid by a less viscous one in a Hele-Shaw cell, the so-called Saffman-Taylor problem [1-5], is a prototypical pattern formation problem. Since the seminal work of Saffman and Taylor [1] a considerable effort has been aimed at understanding both steady and unsteady interfacial patterns formed during this flow. The SaffmanTaylor problem is the simplest member of a wide class of interfacial pattern formation problems such as free dendritic growth, directional solidification, or chemical electrodeposition [6-8]. As such, a theoretical understanding of HeleShaw flow may help elucidate generic behavior common to many pattern forming systems. Despite its relatively simple formulation and the large amount of work devoted to it, however, several aspects of interfacial dynamics in Hele-Shaw flow are still poorly understood, in particular concerning the highly nonlinear and nonlocal dynamics of finger competition.

One of the reasons for the recent interest in Hele-Shaw flow, at least from a mathematical point of view, is that explicit time-dependent solutions can be found in the case of zero surface tension [9-12]. However, it is also known that the zero-surface-tension Saffman-Taylor (ST) problem is ill posed as an initial value problem [13] and finite-time singularities appear frequently [14]. Nevertheless, rather large classes of zero-surface-tension solutions have been found which exhibit the variety of morphologies observed both in experiments and numerical simulations. Then, the question that naturally arises is to what extent smooth (nonsingular) zero-surface-tension solutions reproduce the dynamics of the physical problem with finite surface tension, in particular in the limit of vanishing dimensionless surface tension, $B \rightarrow 0$.

It is well known that surface tension is a singular perturbation to the zero-surface-tension problem [13]. This singular character shows up dramatically in the classical selection problem posed by Saffman and Taylor [1] and only solved three decades later [15-18]. Another manifestation of the singular nature of surface tension which is directly relevant to the present work is its effect on the dynamics. Siegel, Tanveer, and Dai $[19,20]$ showed that interfacial evolution for the regularized problem (i.e., vanishingly small $B$ ) may differ significantly from that for the $B=0$ problem in order one time.

The physical content of exact zero-surface-tension solutions with polelike singularities has been recently addressed in Refs. [5,21,22] using a dynamical systems approach. Through a detailed study it has been shown that the exact zero-surface-tension phase flow, considered in a global sense, is structurally unstable. Consequently, the zerosurface-tension phase dynamics are not topologically equivalent to the phase-space flow of the physical problem, regularized by surface tension. Indeed, the zero-surface-tension phase flow omits the necessary saddle-point structure of multifinger fixed points, which is crucial to the physical finger competition process [22]. A natural extension of the well known solvability mechanism (first applied to "select" a finger of width $1 / 2$ out of a continuum of solutions in the single finger case) was proposed for multifinger solutions in Ref. [22]; this helps clarify how the introduction of surface tension modifies the global phase-space structure of the flow and restores the hyperbolicity of multifinger fixed points.

The approach of Ref. [22], however, was qualitative in nature and could not quantify the extent to which zerosurface-tension trajectories might resemble the evolution with small surface tension. In particular it was recognized that, while some trajectories appear to be qualitatively correct for infinite time, others may have a dramatically different evolution.

A satisfactory analytical understanding of the problem with $B \neq 0$ has been achieved in two regimes: the initial linear instability of the flat interface followed by the weakly nonlinear regime [23], and the asymptotic regime, where sur- 
face tension selects the width of the single finger [15-18]. The highly nonlinear intermediate regime that connects the quasiplanar interface with the asymptotic single-finger regime has mostly been studied through numerical computation [see, e.g., Refs. [24-28]] also combined with qualitative techniques [29,30]. Dai and Shelley [31] showed that for small $B$ numerical computations are extremely sensitive to the precision used in the computations. As a consequence noise level has to be controlled with care in order to ensure that the computation is sufficiently accurate. Computations using very high-precision arithmetic are reported in Refs. $[32,33]$.

An analytical treatment of this highly nonlinear and nonlocal free-boundary problem faces challenging difficulties. In particular, a perturbative study for small $B$ is complicated by the ill posedness of the zero-surface-tension problem. Tanveer [13] was able to overcome this obstacle by embedding the zero-surface-tension problem in a well-posed one. In addition, this well-posed extension of the $B=0$ problem allowed Baker et al. [34] to develop a numerical method to compute the time evolution of zero-surface-tension dynamics in a well-posed manner. Once the $B=0$ problem is formulated in a well-posed way the $B \neq 0$ case can be studied using a perturbative approach. The main result of the asymptotic perturbative theory developed by Tanveer [13] is that the effect of very small surface tension may be significant in a $O$ (1) time. Siegel et al. [20] have extended the work of Ref. [13] to later stages of the evolution, and through numerical computation for very small values of $B$ they confirmed the predictions of the perturbative theory. The zero-surfacetension solutions studied by Siegel et al. $[19,20]$ in the channel geometry were single-finger solutions with an asymptotic width $\lambda$, specifically chosen to be incompatible with selection theory for vanishing surface tension. They found that the singular effect of surface tension was to widen the finger in order to reach the selected width. The surprising feature there was that the effect of surface tension is felt in order-one time, i.e., that the time lapse for which the regularized solution approaches the unperturbed one as $B \rightarrow 0$ is bounded.

The present paper expands the work of Refs. $[19,20]$ in the spirit of Ref. [22], towards the study of multifinger solutions. However, unlike the studies of $[19,20]$ we choose zerosurface-tension multifinger solutions that are compatible with selection theory, to isolate the effects on finger competition from the effects on the selection of the width. We find that the effect of small surface tension on finger competition can be quite dramatic.

The paper is organized as follows. In Sec. II the equations describing Hele-Shaw flow are introduced, and a class of two-finger zero-surface-tension solutions relevant to twofinger competition is presented and briefly discussed. In Sec. III the basic features of the asymptotic theory are recalled, and the theory is applied to the zero-surface-tension solutions introduced in the previous section. The numerical computations with finite (but small) $B$ are presented in Sec. IV. Section V discusses and summarizes the results obtained in previous sections.

\section{ZERO-SURFACE-TENSION}

In this section we present the equations which govern the interfacial dynamics in a rectilinear Hele-Shaw cell, following the formalism of Ref. [13]. We consider a class of exact, time-dependent zero-surface-tension solutions that are relevant to the finger competition problem, and briefly describe the solutions within this class.

Consider Hele-Shaw flow in the channel geometry, in which a fluid of negligible viscosity displaces a viscous liquid. The equations governing the interfacial evolution can be conveniently formulated by first introducing a conformal map $z(\zeta, t)$ which takes the interior of the unit semicircle in the $\zeta$ plane into the region occupied by the viscous fluid in the complex plane $z=x+\mathrm{i} y$, in such a way that the $\operatorname{arc} \zeta$ $=e^{\mathrm{is}}$ for $s \in[0, \pi]$ is mapped to the interface and the diameter of the semicircle is mapped to the channel walls [37] The mapping function $z(\zeta, t)$ has the form $z(\zeta, t)=$ $-(2 / \pi) \ln \zeta+\mathrm{i}+f(\zeta, t)$, and inside and on the unit semicircle we require $f(\zeta, t)$ to be analytic and $z_{\zeta}(\zeta, t) \neq 0$. In addition, we require that $\operatorname{Im} f=0$ on the real diameter of the semicircle. This latter condition ensures that $z$ maps the diameter to the channel walls. Under suitable assumptions [see, Ref. [13]] the Schwartz reflection principle may be applied to show that $f$ is analytic and $z_{\zeta} \neq 0$ for $|\zeta| \leqslant 1$.

The effective velocity field, averaged across the plate gap, is a two-dimensional potential flow satisfying Darcy's law $\mathbf{u}=\boldsymbol{\nabla} \varphi$. Here $\varphi$ is a velocity potential defined by $\varphi=$ $-\left(b^{2} / 12 \mu\right) p$, where $p$ is the pressure, $\mu$ is the viscosity and $b$ is the gap width. Under the assumption of incompressibility $(\boldsymbol{\nabla} \cdot \mathbf{u}=0)$ the potential satisfies Laplace's equation $\nabla^{2} \varphi$ $=0$. Incompressibility also implies the existence of a stream function $\psi$. Therefore, one can define a complex velocity potential $W(z, t)=\varphi+\mathrm{i} \psi$ which is analytic for $z$ in the fluid region of the channel. Its form as a function of $\zeta$ reads

$$
W(\zeta, t)=-(2 / \pi) \ln \zeta+\mathrm{i}+\omega(\zeta, t),
$$

where $\omega(\zeta, t)$ is an analytic function inside the unit circle. In the absence of surface tension, $\omega=0$ [see Eq. (3)].

At the interface we impose the usual boundary conditions. The kinematic boundary condition states that the normal component of fluid velocity at a point on the interface equals the normal velocity of the interface at that point, and takes the form

$$
\operatorname{Re}\left[\frac{z_{t}}{\zeta z_{\zeta}}\right]=\frac{1}{\left|z_{\zeta}\right|^{2}} \operatorname{Re}\left[\zeta W_{\zeta}\right] .
$$

The dynamic boundary condition specifies that the pressure jump across the interface is balanced by surface tension, and is given by

$$
\operatorname{Re} \omega=-\frac{B}{\left|z_{\zeta}\right|} \operatorname{Re}\left[1+\zeta \frac{z_{\zeta \zeta}}{z_{\zeta}}\right] .
$$

The parameter $B$ is the nondimensional surface tension and is defined by $B=b^{2} T / 12 \mu V a^{2}$, where $T$ is the surface tension, $V$ is the fluid velocity at infinity and $a$ is half the cell width. 
Eqs. (1)-(3) are in nondimensional form, with lengths and velocities nondimensionalized by $a$ and $V$, respectively.

When $B=0$ it is well known that pole singularities in $z_{\zeta}$ (i.e., in $f_{\zeta}$ ) present in the exterior of the unit disk are preserved under the dynamics, i.e., such singularities are neither created nor destroyed, although the location of those which are initially present will evolve with time. Exact $B=0$ solutions consisting of a collection of pole singularities with constant amplitude have been the focus of extensive studies [see e.g., Ref. [9]]. The simplest such solution leading to nontrivial finger competition consists of a pair of singularites in the upper halfplane of $|\zeta|>1$, located at positions that are symmetric with respect to the $y$ axis. A second pair of poles conjugate to the first pair is required to satisfy the symmetry restriction $\operatorname{Im} f=0$. This exact solution takes the form $[5,21,22]$

$$
\begin{aligned}
z(\zeta, t)= & -\frac{2}{\pi} \ln \zeta+\frac{1}{\pi}(1-\lambda+i \epsilon) \ln \left(1-\frac{\zeta^{2}}{\zeta_{s}(t)^{2}}\right) \\
& +\frac{1}{\pi}(1-\lambda-i \epsilon) \ln \left(1-\frac{\zeta^{2}}{\bar{\zeta}_{s}(t)^{2}}\right)+d(t)+i,
\end{aligned}
$$

where $\lambda$ and $\epsilon$ are real constants with $0<\lambda<1$ and $\epsilon \geqslant 0$, and $d(t)$ is real. The singularity locations are given by the complex parameter $\zeta_{s}(t)$, which satisfies a simple differential equation given in Ref. [21]. Analyticity of $f(\zeta, t)$ in the unit circle implies that $\left|\zeta_{s}(t)\right|>1$. We employ the convention that $\zeta_{s}(t)$ is a complex number in the first quadrant. The amplitudes of the singularities, given here by the numbers $1-\lambda+i \epsilon$ and its conjugate, are chosen so that the asymptotic form of the solution consists of one or two steadily propagating fingers of total width $\lambda$. The parameter $\epsilon$ determines the nature of the finger competition for $B=0$.

We summarize the features of the solution (4) that are most relevant to the study of finger competition. Consider first $\epsilon=0$. In this case the asymptotic configuration consists of one or two fingers of total width $\lambda$, depending on the initial condition. The singularities move toward the unit disk, with the limit as $t \rightarrow \infty$ denoted by $\zeta_{s}(t) \rightarrow e^{i \theta}$. When $\theta=0$ the asymptotic configuration is a single Saffman-Taylor finger growing in the center of the channel [this asymptotic configuration is denoted ST(R)], for $\theta=\pi / 2$ it is a "side" Saffman-Taylor finger i.e., a pair of half fingers of total width $\lambda$ with tips located at the cell walls [denoted ST(L)], and for $\theta=\pi / 4$ it is a "double" Saffman-Taylor finger, namely two identical fingers of width $\lambda / 2$ with tips at $x=0, \pm 1$ (denoted 2ST). For any other value of $\theta$ the asymptotic configuration consists of two unequal steadily growing fingers, as a consequence of the continuum of fixed points that is present in the phase portrait of the dynamical variables, namely $\left[\operatorname{Re} \zeta_{s}(t), \operatorname{Im} \zeta_{s}(t)\right]$. Therefore, for $\epsilon=0$ the solution (4) does not exhibit finger competition. In addition, it is important to note that the evolution of Eq. (4) with $\epsilon=0$ is free of finitetime singularities, i.e., $z_{\zeta} \neq 0$ in the domain $|\zeta| \leqslant 1$ for all time. In order to correspond to the notation of Ref. [21] introduce the variable $\alpha(t)=\alpha^{\prime}(t)+\mathrm{i} \alpha^{\prime \prime}(t)=1 /\left[i \zeta_{s}^{2}(t)\right]$.
Then the planar interface corresponds to $\alpha=0$; the fixed point $\mathrm{ST}(\mathrm{R})$ to $\alpha=-i$; $\mathrm{ST}(\mathrm{L})$ to $\alpha=i$; and 2ST to $\alpha=1$.

For $\epsilon \neq 0$ the continuum of fixed points is removed, as is the double Saffman-Taylor finger fixed point 2ST. Consequently, the solution to Eq. (4) exhibits "successful" competition, in the sense that the asymptotic interface shape consists of a single Saffman-Taylor finger or side SaffmanTaylor finger. The price to pay is the appearance of finitetime singularities for a certain subset of initial conditions, in the form of a zero of $z_{\zeta}$ impacting the unit disk [this is a generic feature of conformal map solutions $z_{\zeta}$ composed of a finite number of pole singularities-see Ref. [9]]. Then, only the subset of initial conditions free of finite-time singularities is capable of sustaining finger competition all the way to the $t \rightarrow \infty$ outcome. Nevertheless, one may ask whether the class of $B=0$ solutions that are free of finite-time singularities may describe, at least qualitatively, the physical finger competition for positive surface tension in the limit $B \rightarrow 0$.

\section{ASYMPTOTIC THEORY}

Little is known about the effect of finite (but small) surface tension $B$ on the dynamics of zero-surface-tension multifinger solutions, and in particular on the class of exact solutions (4). For single-finger configurations, steady state selection theory predicts that the finger cannot have an arbitrary width. Indeed, for vanishing surface tension $B \rightarrow 0$ the width $\lambda=1 / 2$ is selected, asymptotically in time. Thus, it is clear that surface tension has a critical influence on singlefinger solutions with $\lambda \neq 1 / 2$, as was shown by Siegel, Tanveer, and Dai $[19,20]$.

Consider now the effect of small surface tension on the exact $(B=0)$ two-finger solution (4). When $0<B \ll 1$ the asymptotic perturbation theory developed in Refs. $[19,13,20]$ can be applied. This perturbation theory describes the effects of the introduction of a small amount of surface tension on initial data $z(\zeta, 0)$ specified in the extended complex plane, i.e., in a domain including the "unphysical" region $|\zeta|>1$ (the extended domain is required to make the $B=0$ problem well posed). The effect of finite $B$ is most important near isolated zeros and singularities of $z_{\zeta}(\zeta, 0)$, where a regular perturbation expansion in $B$ breaks down. For the class of solutions (4) we are discussing, the isolated singularities of $z_{\zeta}(\zeta, 0)$ are simple poles. The theory suggests that the introduction of finite surface tension modifies the poles $\left(\zeta_{s}\right)$ by transforming them into localized clusters of $-4 / 3$ singularities, but this has no significant influence on the interfacial shape.

The influence of surface tension on the zeros of $z_{\zeta}(\zeta, 0)$ is more complex. Each initial zero instantly gives birth to two localized inner regions, i.e., regions where the $B=0$ and $B>0$ solutions differ by $O(1)$ (the theory predicts that these inner regions also contain clusters of $-4 / 3$ singularities). One of the two inner regions moves, at least initially, according to the $B=0$ dynamics of the original zero $\zeta_{0}$ [38], and has a negligible influence on the interface in the case we study. The second inner region created around $\zeta_{0}(0)$ moves differently: to leading order in $B$ it moves like a singularity of the zero-surface-tension problem and this speed is differ- 
ent form the speed of the zero $\zeta_{0}(t)$, which spawned the cluster. As this singularity cluster approaches the physical domain it may perturb the flow and the interface shape may differ significantly from that at $B=0$ shape. The location of this singularity cluster will be denoted by $\zeta_{d}(t)$, and following Ref. [13] we shall call it the daughter singularity. We emphasize that the dynamics of the daughter singularity is determined at lowest order solely by the $B=0$ solution $z^{0}(\zeta, t)$, at least until it arrives at the surroundings of the unit circle, and therefore can be simply computed once the initial locations of the zeros of $z_{\zeta}(\zeta, 0)$ are determined.

The daughter singularity evolution equation is given by (see Ref. [13])

$$
\dot{\zeta}_{d}(t)=-q_{1}^{0}\left(\zeta_{d}(t), t\right) ; \quad \zeta_{d}(0)=\zeta_{0}(0),
$$

where $q_{1}^{0}$ is defined by

$$
q_{1}^{0}=\frac{\zeta}{2 \pi i} \oint_{\left|\zeta^{\prime}\right|=1} \frac{d \zeta^{\prime}}{\zeta^{\prime}} \frac{\zeta+\zeta^{\prime}}{\zeta^{\prime}-\zeta} \frac{\operatorname{Re}\left[\zeta^{\prime} W_{\zeta}^{0}\left(\zeta^{\prime}, t\right)\right]}{\left|z_{\zeta}^{0}\left(\zeta^{\prime}, t\right)\right|^{2}}
$$

and the superscript zero denotes that the function evaluations are done using the corresponding $B=0$ solution. The function $-q_{1}^{0}(\zeta, t)$ also gives the characteristic velocity of a pole or branch point singularity of $z_{\zeta}(\zeta, t)$ located at position $\zeta$ in the region $|\zeta|>1$. The daughter singularity approaches the unit circle [13] and it can impact it in a finite time $t_{d}$, the daughter singularity impact time, satisfyng $\left|\zeta_{d}\left(t_{d}\right)\right|=1$. In the limit $B \rightarrow 0$, the daughter singularity impact time $t_{d}$ signals the time when the effects of the surface tension are felt on the physical interface. For times larger than $t_{d}$ the $B=0$ interface and the $B \rightarrow 0$ are expected to differ significantly.

For the family of exact $B=0$ solutions the mapping function (4) has four polelike singularities: $\pm \zeta_{s}$ and $\pm \bar{\zeta}_{s}$, and four zeros $\pm \zeta_{0+}$ and $\pm \zeta_{0-}$ of $z_{\zeta}$ located at

$$
\begin{aligned}
\zeta_{0+}^{2}= & \frac{-(\lambda+i \epsilon) \zeta_{s}^{2}-(\lambda-i \epsilon) \bar{\zeta}_{s}^{2}}{2(1-2 \lambda)} \\
& +\frac{\sqrt{\left[(\lambda+i \epsilon) \zeta_{s}^{2}+(\lambda-i \epsilon) \bar{\zeta}_{s}^{2}\right]^{2}+4(1-2 \lambda)\left|\zeta_{s}\right|^{4}}}{2(1-2 \lambda)}
\end{aligned}
$$

$$
\begin{aligned}
\zeta_{0-}^{2}= & \frac{-(\lambda+i \epsilon) \zeta_{s}^{2}-(\lambda-i \epsilon) \bar{\zeta}_{s}^{2}}{2(1-2 \lambda)} \\
& -\frac{\sqrt{\left[(\lambda+i \epsilon) \zeta_{s}^{2}+(\lambda-i \epsilon) \bar{\zeta}_{s}^{2}\right]^{2}+4(1-2 \lambda)\left|\zeta_{s}\right|^{4}}}{2(1-2 \lambda)} .
\end{aligned}
$$

For the particular case $\lambda=1 / 2$ this solution presents only one pair of zeros $\pm \zeta_{0}$ located at

$$
\zeta_{0}^{2}=\frac{\left|\zeta_{s}\right|^{4}}{2\left[(\lambda+i \epsilon) \zeta_{s}^{2}+(\lambda-i \epsilon) \bar{\zeta}_{s}^{2}\right]} .
$$

In the following it will be useful to define the real quantity $\beta=-(\lambda+i \epsilon) \zeta_{s}^{2}-(\lambda-i \epsilon) \bar{\zeta}_{s}^{2}$ which appears in Eqs. (7) and (8).

Depending on the value of $\lambda$ the initial data may have zeros on both the real and imaginary axes, or all the zeros may lie on a single axis. This difference has significant consequences in the finite surface tension dynamics. More specifically, when $\lambda<1 / 2$ the zeros described in Eqs. (7a) and (7b) are located on both the real and imaginary axes of $|\zeta|$ $>1$, namely at $\pm\left|\zeta_{0+}\right|$ and $\pm i\left|\zeta_{0-}\right|$. The situation is different for $\lambda>1 / 2$, which is further divided into two cases, depending on whether $\beta^{2}+4(1-2 \lambda)\left|\zeta_{s}\right|^{2}>0$ or $<0$. In the former case all four singularities lie on the real axis (for $\beta$ $>0$ ) or on the imaginary axis (for $\beta<0$ ). In the latter case the four zeros are located off the axes in conjugate pairs, i.e., at $\pm \zeta_{0}$ and $\pm \bar{\zeta}_{0}$. Finally, when $\lambda=1 / 2$ the solution (4) has only two zeros, located on the real axis at $\pm\left|\zeta_{s}\right|^{2} / \sqrt{-2 \beta}$ when $\beta<0$ and on the imaginary axis at $\pm\left|\zeta_{s}\right|^{2} / \sqrt{2 \beta}$ when $\beta>0$. Note that for $\lambda=1 / 2$ the $B=0$ solution has two less zeros than for $\lambda \neq 1 / 2$.

The initial zero locations described above have a critical bearing on whether the daughter singularity will impact the unit disk [39]. Although all daughter singularities approach the unit disk, their impact may be shielded by the presence of an inner region corresponding to a pole singularity. More precisely, since $\zeta_{d}$ and $\zeta_{s}$ obey the same dynamical equation, they will move together if they get close enough to each other. However, the inner region around a pole moves to leading order like the $B=0$ pole, i.e., it moves exponentially slowly toward $|\zeta|=1$ when $\left|\zeta_{s}\right|-1 \ll 1$, and does not impinge upon the unit disk in finite time [9]. In this case the $O\left(B^{1 / 3}\right)$ inner region around the daughter singularity will not affect the dynamics on $|\zeta|=1$, at least until $t=O(-\ln B)$. Before this time, we expect the interface to be uninfluenced by the presence of the daughter singularity. This shielding mechanism is discussed in the context of single fingers in Ref. [20].

Knowledge of the $t \rightarrow \infty$ asymptotic state and the initial locations of zeros can be used to ascertain whether shielding can occur. The $B=0$ asymptotic state corresponds to $\zeta_{s}^{2}(t$ $\rightarrow \infty) \rightarrow \pm 1$. Thus, for $\lambda<1 / 2$, only one pair of daughter singularities may be shielded-never both - so at least one pair of daughter singularities will impinge on the unit disk. The daughter singularities will also not be shielded when $\lambda$ $>1 / 2$ and $\beta^{2}+4(1-2 \lambda)\left|\zeta_{s}\right|^{2}<0$. However, for $\lambda>1 / 2$ and $\beta^{2}+4(1-2 \lambda)\left|\zeta_{s}\right|^{2}>0$ it is possible for all the daughter singularities to be shielded, since they lie on a single axis. The daughter singularities can also be completely shielded when $\lambda=1 / 2$. The different possibilities are schematically depicted in Fig. 1.

We have numerically computed the daughter singularity impact time $t_{d}$ for various values of $\lambda$ and $\epsilon$, using initial conditions close to the planar interface, $\left|\zeta_{s}\right|^{2}=20$ and various values of $\operatorname{Arg}\left[\zeta_{s}^{2}\right]$. Figure 2 shows the phase portrait for different values of $\lambda$ and $\epsilon$ with the daughter singularity impact indicated. From the plots it is immediately seen that for $\lambda<1 / 2$ at least one daughter singularity always hits the unit circle, and for $\lambda \geqslant 1 / 2$ some trajectories are free from 
(a)

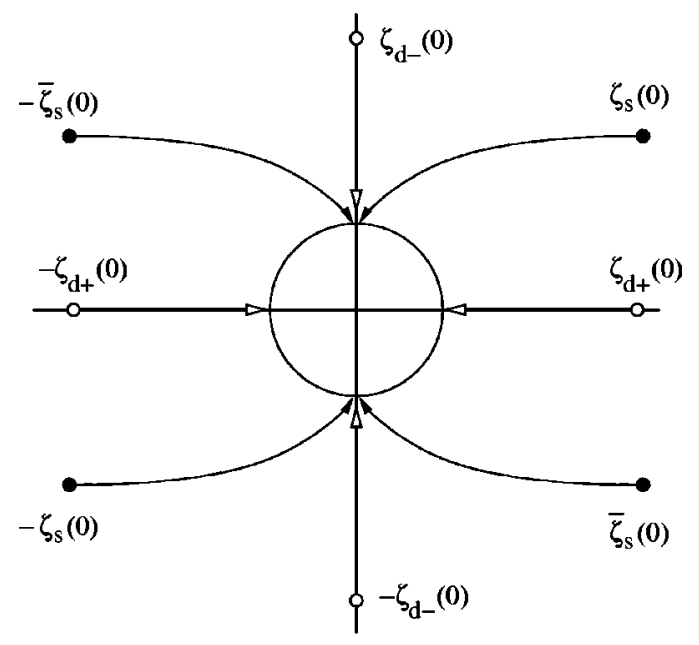

(b)

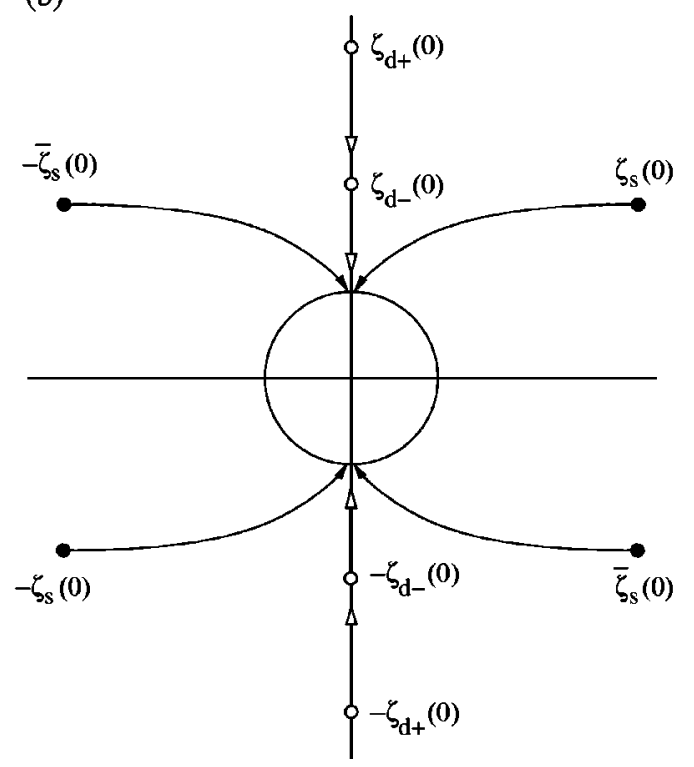

FIG. 1. (a) Schematic representation of the dynamics of pole $\zeta_{s}$ and daughter $\zeta_{d}$ singularities for $\lambda<1 / 2$. (b) Schematic representation of one of the two possible dynamics of pole $\zeta_{s}$ and daughter $\zeta_{d}$ singularities for $\lambda>1 / 2$.

daughter singularity impact. In addition, it is observed that for fixed $\lambda$ a larger value of $\epsilon$ causes the daughter singularities to hit in shorter times (or less developed fingers) than a smaller value of $\epsilon$, and for fixed $\epsilon$ larger $\lambda$ implies larger impact times. We have also checked that the daughter singularity impact occurs well before a finite-time singularity, i.e., the impact of a zero of $z_{\zeta}$. Thus, the effect of surface tension is significant well before the curvature in the zero-surfacetension solution becomes large.

It is noted that the $\lambda$ dependence of the daughter singularity impact is consistent with the results of steady state selection theory [15-18]. According to selection theory, for small $B$ the possible values of $\lambda$ are discretized: $\lambda$ must satisfy the relation $\lambda=\lambda_{n}(B)$, given to leading order by

$$
\lambda_{n}(B)=\frac{1}{2}\left\{1+\left(\frac{1}{8} \pi^{2} C_{n} B\right)^{2 / 3}\right\} \quad n=0,1,2, \ldots,
$$

(a)

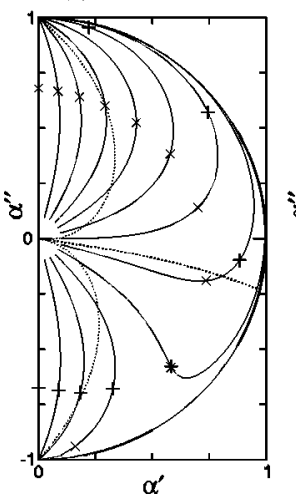

(b)

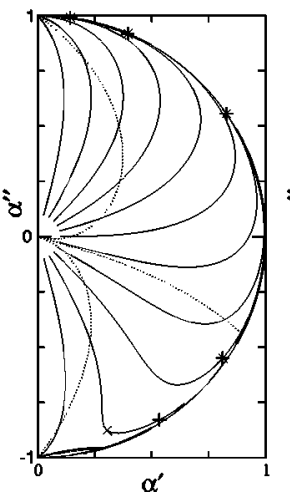

(c)

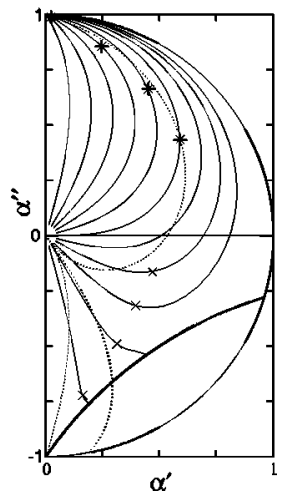

FIG. 2. Phase portraits for (a) $\lambda=1 / 3$ and $\epsilon=0.1$, (b) $\lambda=2 / 3$ and $\epsilon=0.1$, and (c) $\lambda=1 / 3$ and $\epsilon=1 / 2$. The daughter singularity impact is indicated by the symbols. The + symbol corresponds to the impact of $\zeta_{d+}, \times$ to the impact of $\zeta_{d_{-}}$, and $*$ to the simultaneous impact of $\zeta_{d+}$ and $\zeta_{d-}$.

where $n$ parametrizes the branch of solutions. Note that $\lambda_{n}$ $>1 / 2$ for all $n$. The steady finger shape is to leading order a Saffman-Taylor finger, with the above values of $\lambda_{n}$ substituted for the width $\lambda$. On the other hand, for $\epsilon>0$ the asymptotic state of Eq. (4) is a Saffman-Taylor finger of width $\lambda$. From Eq. (9) it is clear there exists a steady solution with width $\lambda_{n}(B)$ close to a Saffman-Taylor finger of arbitrary width $\lambda>1 / 2$. Thus the shielding of the daughter singularity, which leads to the persistence of a Saffman-Taylor solution with $\lambda>1 / 2$ over long times, is consistent with steady state selection theory [40]. In contrast for $\lambda<1 / 2$ there are no nearby steady solutions. Thus, a Saffman Taylor finger with $\lambda<1 / 2$ cannot persist over a long time. We see that the impact of a daughter singularity provides a mechanism for the onset of finger competition, finger widening, and selection of a width $\lambda>1 / 2$.

For $\epsilon=0$ the scenario is similar, except there is an added class of exact $B>0$ solutions. Magdaleno and Casademunt [35] have shown that two-finger solutions composed of steadily propagating but unequal fingers do exist for small nonzero $B$. The introduction of a small nonzero surface tension selects a discrete set of solutions from the continuum of fixed points of the $B=0$ phase portrait. The solutions are parametrized by the total width of the fingers $\lambda=\lambda_{1}+\lambda_{2}$ and the relative width $q=\lambda_{1} / \lambda$, and the introduction of finite $B$ discretizes the possible values of the parameters. In particular, they must satisfy a condition of the form $\lambda=\lambda_{n}(B)$ and $q=q_{n, m}(B)$, where $n$ and $m$ are integers. The expression for $\lambda_{n}(B)$ at lowest order is equivalent to Eq. (9), but with different coefficients $C_{n}$. The shape of these solutions are given to leading order (in the limit $t \rightarrow \infty$ ) by Eq. (4) with allowed value of $\lambda_{n}(B)$ substituted for the width $\lambda$. Again, $\lambda_{n}(B)$ $>1 / 2$, and the consistency between daughter singularity impacts and steady state selection theory follows as above.

We conjecture that the outcome of interfacial shape evolution after the daughter singularity impinges is in general independent of the particular finger on which the impact first occurs i.e., independent of the point at which $\zeta_{d}(t)$ impacts on $|\zeta|=1$. More specifically, we surmise that impact on ei- 
ther the shorter (trailing) or larger (leading) finger retards the velocity of that finger, and is accompanied by the widening of the leading finger, so as to maintain a constant fluid flux at infinity. The widened leading finger then shields the trailing finger, preventing it from further growth. Thus, the finger that is leading at the time of the daughter singularity impact "wins" the competition, in the sense that it will evolve for $t \rightarrow \infty$ to the ST steady finger. To examine this conjecture and study the dynamics of finger competition with finite (but small) surface tension we have numerically computed the evolution of an interface with initial conditions given by Eq. (4). The results are reported in the following section.

\section{NUMERICAL RESULTS}

Numerical computations have been performed for $B>0$, using an initial interface corresponding to the explicit $B=0$ solutions discussed in Sec. II. The effect of positive surface tension on this class of solutions is explored for various values of $\epsilon$ and a variety of initial pole positions. To isolate the effects inherent to finger competition from those of width selection, we will concentrate on $B=0$ solutions with $\lambda$ $=1 / 2$, the value selected by surface tension in the limit $B \rightarrow 0$.

We employ the numerical method introduced by Hou et al. [28] and used in other studies of small surface tension effects in Hele-Shaw flow $[19,20,32]$. The method is described in detail in Ref. [28]. It is a boundary integral method in which the interface is parametrized at equally spaced points by means of an equal-arclength variable $\alpha$. Thus, if $s(\alpha, t)$ measures arclength along the interface then the quantity $s_{\alpha}(\alpha, t)$ is independent of $\alpha$ and depends only on time. The interface is described using the tangent angle $\theta(\alpha, t)$ and the interface length $L(t)$, and these are the dynamical variables instead of the interface $x$ and $y$ positions. The evolution equations are written in terms of $\theta(\alpha, t)$ and $L(t)$ in such a way that the high-order terms, which are responsible of the numerical stiffness of the equations, appear linearly and with constant coefficients. This fact is exploited in the construction of an efficient numerical method, i.e., one that has no time step constraint associated with the surface tension term yet is explicit in Fourier space. We have used a linear propagator method that is second order in time, combined with a spectrally accurate spatial discretization. Results in this section are specified in terms of the scaled variables

$$
\tilde{t}=\pi t, \quad \widetilde{B}=\pi^{2} B, \quad \tilde{x}=\pi x, \quad \tilde{y}=\pi y,
$$

instead of the original ones used in previous sections.

The number of discretization points is chosen so that all Fourier modes of $\theta(\alpha, t)$ with amplitude greater than roundoff are well resolved, and as soon as the amplitude of the highest-wave-number mode becomes larger than the filter level the number of modes is increased, with the amplitude of the additional modes initially set to zero. The time step $\Delta t$ is decreased until an additional decrease does not change the solution to plotting accuracy, nor lead to any significant differences in any quantities of interest. In a typical calculation 512 discretization points are initially used, and the initial time step is $\Delta t=5 \times 10^{-4}$. For small values of surface tension numerical noise is a major problem, and the spurious growth of short-wavelength modes induced by roundoff error must be controlled. To help prevent this noise-induced growth at short-wavelengths spectral filtering [36] is applied. Additionally, we minimize noise effects and also assess the time at which these effects become prevalent by employing extended precision calculations, as described in the following section.

\section{A. Solutions with $\epsilon=0$}

We first consider parameter values $\lambda=1 / 2$ and $\epsilon=0$. A typical set of interfacial profiles is shown in Fig. 3. The initial data is given by the mapping function Eq. (4), with $\lambda=1 / 2, \epsilon=0, d(0)=0$, and $\zeta_{s}^{2}(0)=20 \exp (i \pi / 6)$. With this value of $\zeta_{s}^{2}(0)$ the initial interface is well inside the linear regime. Evolutions are shown for different values of $\widetilde{B}$, and the $B=0$ interface evolution is also plotted for comparison. In all these evolutions the filter level is set to $10^{-13}$, although later we shall make comparisons to profiles computed at higher precision.

For the largest value of surface tension the computed $B>0$ and the exact $B=0$ solutions first differ appreciably at the seventh curve, corresponding to $\tilde{t} \approx 3$. At this point the velocity of the small finger (at the channel sides) begins to decrease and it is clearly left behind when compared with the small finger evolution in the $B=0$ solution. Eventually, the advance of the small finger is completely suppressed and the larger finger widens to attain a width close to $1 / 2$ of the channel, the width singled out by selection theory for vanishing $B$. For a smaller value of surface tension, for instance $\widetilde{B}=0.001$, the evolution displays qualitatively the same behavior. The $B>0$ interface differs appreciably from the $B=0$ sightly later than before (i.e., at the eighth curve) and the region where the two solutions differ most is to some extent more localized around the small finger than for larger values of $B$. Additionally, for this value of surface tension the effect of numerical noise is clearly exhibited in the interfacial profiles. Here the tip-splitting and side-branching activities are a clear effect of numerical noise, as can be easily checked redoing the computation with a different noise filter level.

In order to suppress or delay the branching induced by numerical noise that appears for small values of surface tension it is necessary to use higher precision arithmetic, e.g. quadruple precision (128-bit arithmetic). The filter level can then be reduced by a large amount and the outcome of spurious oscillations is substantially delayed. Figure 4 shows the effect of reducing the filter level to $10^{-27}$. The $B=0$ solution is plotted, as well as the computation with double precision. For $\widetilde{B}=0.001$ the branching is totally suppressed, at least for the times we have computed, but for smaller values of $\widetilde{B}$ the use of quadruple precision is only able to delay the branching and not totally suppress it. The quadruple precision computation confirms the results observed with lower precision, the introduction of finite (but small) surface tension results in the suppression of the small finger. From Fig. 4 one can also 
(a)
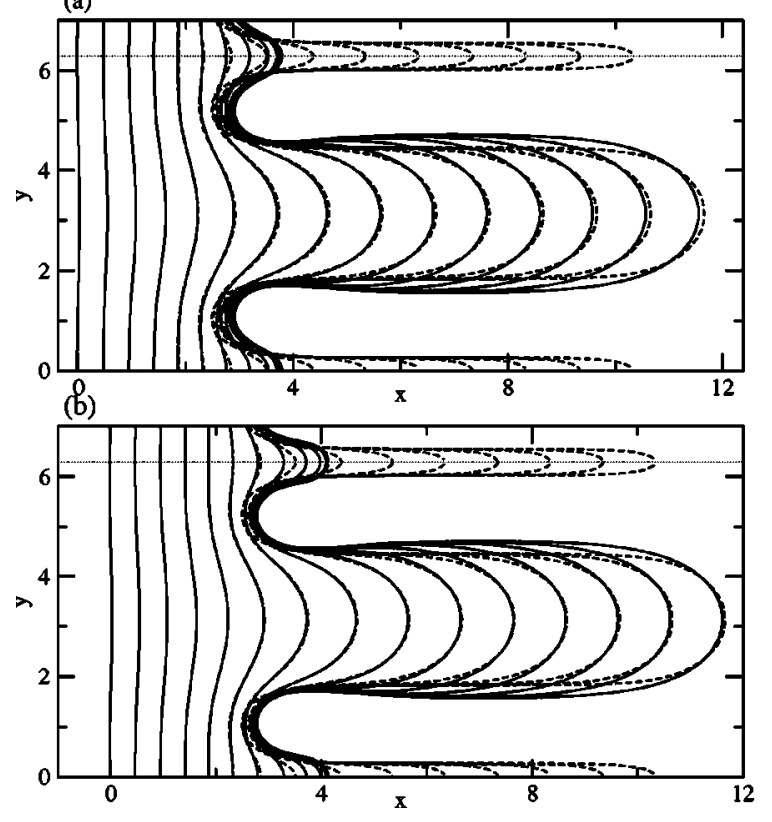

(c)
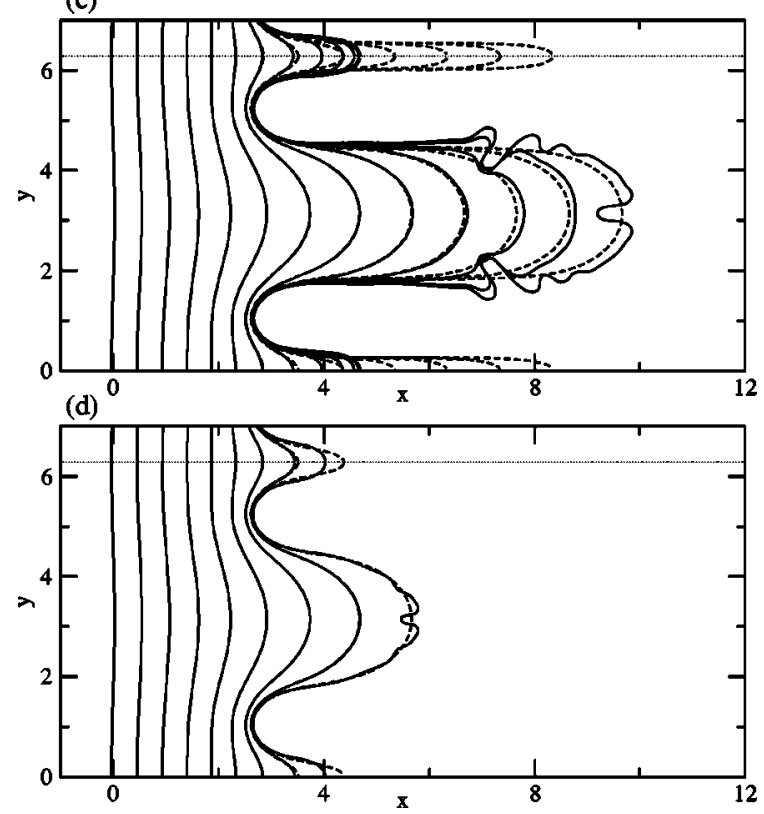

FIG. 3. Evolution of an initial condition of the form (4) with $\lambda=1 / 2, \epsilon=0$, and $\zeta_{s}^{2}(0)=20 \exp (i \pi / 6)$. The solid lines correspond to surface tension $\widetilde{B}$ values (a) 0.01 , (b) 0.005 , (c) 0.001 , and (d) 0.0005 . The dashed lines correspond to the zero-surface-tension evolution. The time difference between different curves is 0.5 . The physical channel in the $y$ direction extends from the origin to the dotted line, and the region above is plotted for better visualization of the lateral finger.

see that for long times, when the interface is clearly affected by numerical noise (in the double precision curve), the noiseinduced branching is restricted to the large finger, and the small finger is basically unaffected by noise. This observation suggests that the small finger shape, as well as its tip velocity and tip curvature, can be trusted even when the large finger has developed tip splittings and side branchings due to the spurious growth of roundoff error.

Figure 5 shows the tip velocity of both fingers versus $\tilde{t}$ for decreasing values of surface tension. It can be seen that the

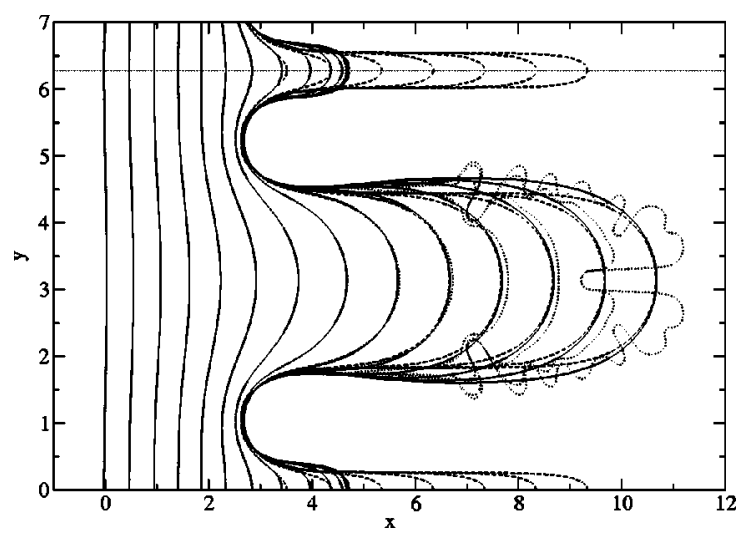

FIG. 4. Evolution of an initial condition of the form (4) with $\lambda=1 / 2, \epsilon=0$, and $\zeta_{s}^{2}(0)=20 \exp (i \pi / 6)$. The solid lines correspond to $\widetilde{B}=0.001$ with a filter level equal to $10^{-27}$, the dotted line corresponds to the same $\widetilde{B}$ but with the filter level equal to $10^{-13}$, and the dashed line corresponds to the zero-surface-tension solution. The time difference between curves is 0.5. As in Fig. 3, the physical channel in the $y$ direction extends from the origin to the dotted line. velocity of the large finger is only slightly affected by surface tension, whereas the velocity of the small finger is substantially reduced by the inclusion of finite $B$. As $\widetilde{B}$ is decreased the tip velocity of the small finger is more faithful to
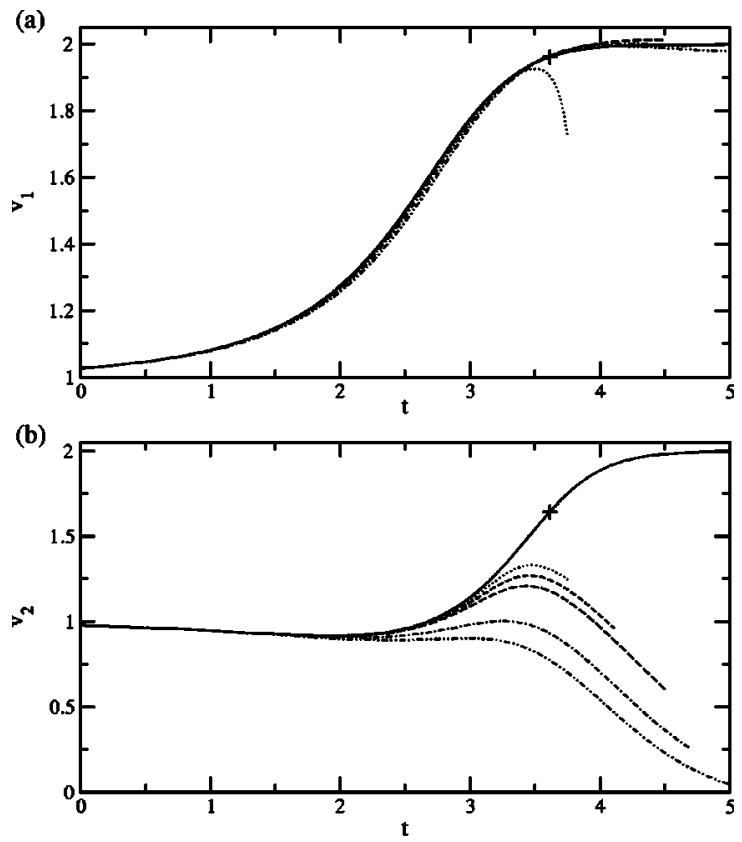

FIG. 5. Computed tip velocities for the initial condition of Fig. 4: (a) corresponds to the central (large) finger and (b) to the lateral (small) finger. The daughter singularity impact time $\tilde{t}_{d}$ is indicated by the + symbol. The value of $\widetilde{B}$ is: 0 (solid line), 0.0002 (dotted line), 0.0005 (dashed line), 0.001 (long dashed line), 0.005 (dotdashed line), and 0.01 (dot-dot-dashed line). 


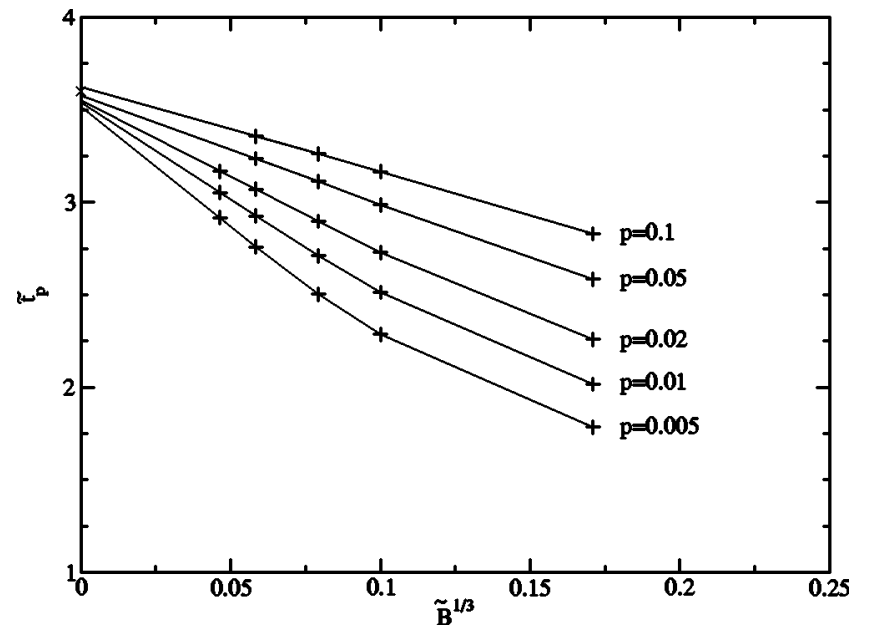

FIG. 6 . The time $\tilde{t}_{p}$ (defined in the text) versus $\widetilde{B}^{1 / 3}$. From top to bottom, $p=0.1,0.05,0.02,0.01,0.005$. The daughter singularity impact time $\tilde{t}_{d}$ is indicated by a $\times$ symbol, and the curves are linearly extrapolated for comparison.

the $B=0$ evolution before the daughter singularity impact (shown by a cross), and clearly veers away from the $B=0$ velocity later in the evolution, consistent with asymptotic theory. Note that at the smallest value of $\widetilde{B}$ the tip velocity of the large finger drastically differs from the $B=0$ velocity at late times. This discrepancy is a manifestation of noise effects in the neighborhood of the large finger tip. However, as previously seen, the small finger is basically unaffected by noise at the times we have plotted.

In order to further verify that the daughter singularity impact is responsible for the observed change in the small finger tip speed we follow the scheme introduced in Ref. [20]. Define $t_{p}$ as the time when the computed tip velocity differs by $p$ from the $B=0$ tip velocity. According to asymptotic theory [13] this $t_{p}$ will be a linear function of $B^{1 / 3}$ in the limit $B \rightarrow 0$, as long as $p$ is small enough. Figure 6 shows $\tilde{t}_{p}$ versus $\widetilde{B}^{1 / 3}$ for various values of $p$, and it can be seen that $\widetilde{t}_{p}$ exhibits the predicted behavior. Moreover, we have extrapolated the $B=0$ value of $\tilde{t}_{p}$ using the two points of lowest $\widetilde{B}$ and the result is very close to $\tilde{t}_{d}$, whose value is represented by a $\times$ symbol. We conclude that the impact of the daughter singularity is associated with the dramatic change of the $B$ $>0$ solution when compared to the zero-surface-tension solution, reducing the velocity of the small finger and eventually suppressing it. In contrast, for the $B=0$ dynamics the small finger "survives," propagating with the same asymptotic speed as the larger finger. Note that the average interface advances at unit velocity, and a tip velocity below one implies that the finger is retreating in the reference frame of the average interface.

It is noted that for the initial condition we have studied the daughter singularity impact takes place on the tip of the small finger. Therefore, the influence of surface tension on the interface should be significant first around the impact point, that is, the small finger tip. Our numerical results show that in fact this is the case; the initial effect of the daughter singularity impact is to slow and then completely stop the growth of the small finger. Later on, as the singularity cluster centered in $\zeta_{d}$ spreads over the unit circle, the effect of surface tension is felt by the whole interface and the large finger widens.

We have also studied the finite surface tension dynamics for a more general class of initial conditions. More precisely, we have studied initial conditions of the form $\zeta_{s}^{2}(0)$ $=20 \exp (i n \pi / 12)$, where $n=0, \pm 1, \ldots, \pm 6$, and have obtained the same qualitative results as in the case previously studied, namely that the presence of small surface tension suppresses the growth of the finger which is trailing at the time of daughter singularity impact. In order to compare the $B=0$ and the $B \neq 0$ dynamics in a compact and global way we have plotted the phase portrait for $B=0$ using the the tip velocities $v_{1}, v_{2}$ as dynamical variables. In the laboratory frame they read

$$
\begin{aligned}
& v_{1}=\frac{1+\mathrm{i}\left(\zeta_{s}^{2}-\bar{\zeta}_{s}^{2}\right)+\zeta_{s}^{2} \bar{\zeta}_{s}^{2}}{\zeta_{s}^{2} \bar{\zeta}_{s}^{2}+\mathrm{i}\left(\zeta_{s}^{2}-\bar{\zeta}_{s}^{2}\right) / 2}, \\
& v_{2}=\frac{1-\mathrm{i}\left(\zeta_{s}^{2}-\bar{\zeta}_{s}^{2}\right)+\zeta_{s}^{2} \bar{\zeta}_{s}^{2}}{\zeta_{s}^{2} \bar{\zeta}_{s}^{2}-\mathrm{i}\left(\zeta_{s}^{2}-\bar{\zeta}_{s}^{2}\right) / 2} .
\end{aligned}
$$

Now a comparison between dynamics for $B=0$ and $B \neq 0$ is straightforward since the trajectories can be plotted together and compared. In addition, the tip velocity is a useful variable because it contains geometric information; specifically the inverse of the tip velocity is equal to the width of the finger in the asymptotic $(t \rightarrow \infty)$ regime. It is important to note that $\left(v_{1}, v_{2}\right)$ are dynamical variables for the $B=0$ problem, so that the plot of the zero-surface-tension trajectories onto the space $\left(v_{1}, v_{2}\right)$ is a true phase portrait. On the other hand $\left(v_{1}, v_{2}\right)$ are not state variables of the problem with finite surface tension, so in this case we simply obtain a projection onto the $\left(v_{1}, v_{2}\right)$ space of the original $B \neq 0$ trajectory, which is embeded in the infinite-dimensional phase space of interface configurations.

Figure 7 shows the phase portrait for $B=0$ together with the tip velocities obtained from the initial conditions described above for $\widetilde{B}=0.01$. From the figure it is evident that the introduction of finite surface tension has substantially changed the global phase dynamics of the problem. Only one $\widetilde{B}=0.01$ trajectory connects the planar interface $(1,1)$ and the 2 ST point $(2,2)$, corresponding to the unsteady double Saffman-Taylor finger. Any other $\widetilde{B}=0.01$ trajectory ends in one of the two ST finger points, ST(L) at $(2,0)$ and ST(R) at $(0,2)$. In contrast, the $(2,2)$ point, equivalent to the continuum of fixed points present with the $\left(\alpha^{\prime}, \alpha^{\prime \prime}\right)$ or $\left(\operatorname{Re} \zeta_{s}, \operatorname{Im} \zeta_{s}\right)$ variables, has a finite basin of attraction for $B=0$. The introduction of finite surface tension has dramatically changed the zero-surface-tension $\left(v_{1}, v_{2}\right)$ trajectories, to the extent that the $B=0$ phase portrait and the $B \neq 0$ projection are not topologically equivalent. This result is not a complete surprise, since it was anticipated from the structural instability of the dynamical system governing the evolution of Eq. (4) for $\epsilon=0$ [21]. A more dramatic example of topo- 


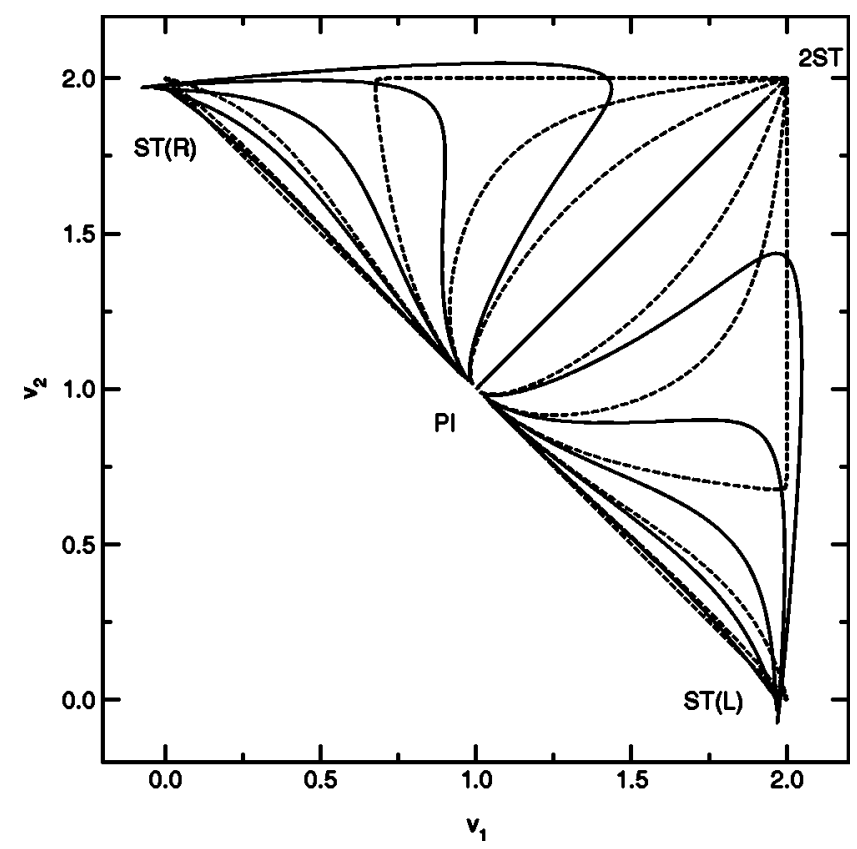

FIG. 7. Plot of the evolution of initial conditions of the form (4) with $\lambda=1 / 2, \quad \epsilon=0, \quad$ and $\zeta_{s}^{2}(0)=20 \exp ($ in $\pi / 12)$ and $n=0$, $\pm 1, \ldots, \pm 6$ in the $\left(v_{1}, v_{2}\right)$ or tip speed space. The solid line corresponds to $\widetilde{B}=0.01$ and the dashed line to $\widetilde{B}=0$.

logical inequivalence of phase portraits will be given in the following section, when we consider the case $\epsilon \neq 0$.

Although the use of the variables $\left(v_{1}, v_{2}\right)$ has allowed us to project the finite surface dynamics onto the zero-surface-

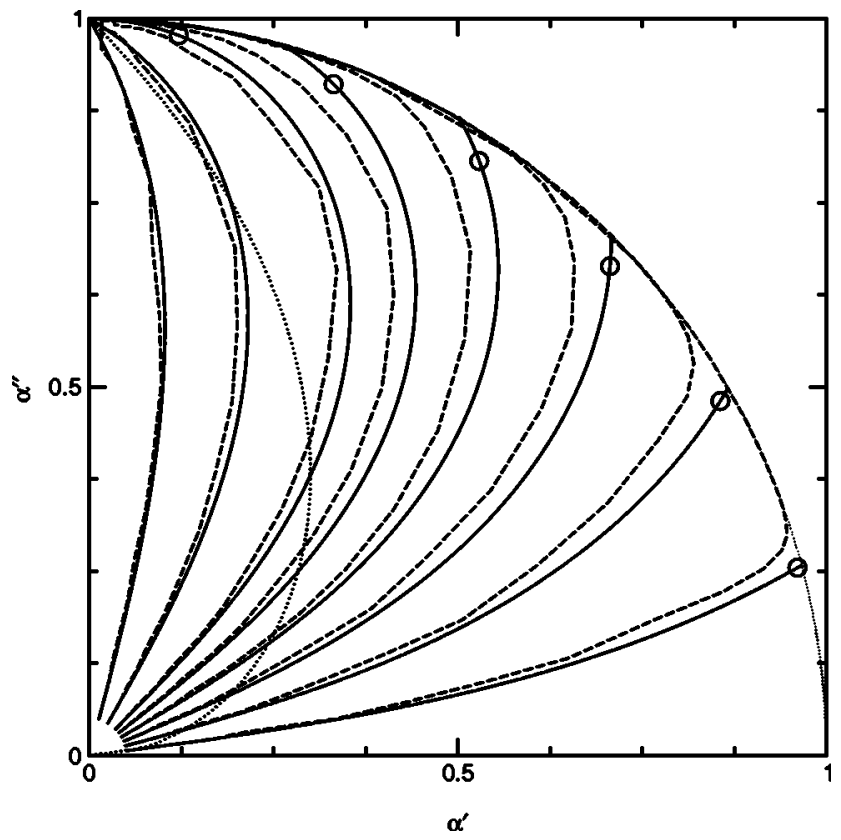

FIG. 8. Comparison between the $\widetilde{B}=0$ trajectories and the projected evolutions with $\widetilde{B}=0.01$, for the initial conditions of Fig. 7 . The solid line corresponds to $\widetilde{B}=0$ and the dashed line to the projection of the $\widetilde{B}=0.01$ evolutions. The daughter singularity impacts are indicated by a circle.

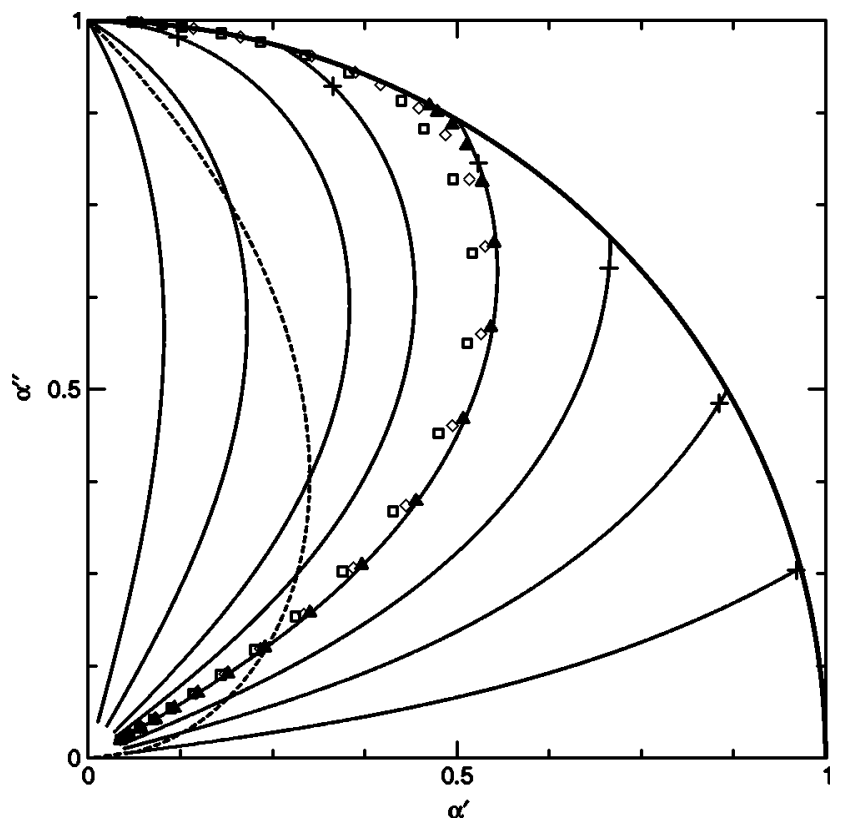

FIG. 9. Comparison between the $\widetilde{B}=0$ trajectories and the projection of the evolution of the initial condition given by Eq. (4) with $\lambda=1 / 2, \epsilon=0$, and $\zeta_{s}^{2}(0)=20 \exp (i \pi / 6)$, where $\boldsymbol{\Delta}$ corresponds to $\widetilde{B}=0.001, \diamond$ to $\widetilde{B}=0.005, \square$ to $\widetilde{B}=0.01$, and $\times$ to $\widetilde{B}=0$. The daughter singularity impacts are indicated by a plus.

tension phase portrait this projection has one major limitation: it only considers a local quantity, the tip velocity. We have also considered a projection that takes more global properties of the interface into account. Specifically, given a computed $B \neq 0$ solution for an initial condition of the form (4), one can use a suitable norm to define a "distance" between the computed interface and the $B=0$ interface obtained from the mapping function Eq. (4). We choose this "distance" to be the area enclosed between the two interfaces at a given time. Additionally, we define a projection of the $B \neq 0$ interface onto the $B=0$ phase space [with phase space variables $\left.\left(\operatorname{Re} \zeta_{s}, \operatorname{Im} \zeta_{s}\right)\right]$ by selecting the value of $\zeta_{s}$ that minimizes the "distance" between the two interfaces, with the restriction that the position of the two mean interfaces must be the same. The latter condition ensures that the projection satisfies mass conservation.

Figure 8 shows the $B=0$ phase portrait and the corresponding projected evolution for surface tension $\widetilde{B}=0.01$. Again, the plot clearly shows that the introduction of finite surface tension modifies the phase portrait of $B=0$. The projected trajectories are initially close to the $B=0$ dynamics, but for well developed fingers (corresponding to $|\alpha| \sim 1$ ) the projection departs from the $B=0$ trajectory towards the Saffman-Taylor fixed point, located at $\alpha^{\prime}=0, \alpha^{\prime \prime}=1$. The projected trajectory only remains close to the corresponding $B=0$ trajectory when the latter evolves towards the Saffman-Taylor fixed point. More precisely, the continuum of fixed points present for $B=0$ has been removed by surface tension and the Saffman-Taylor fixed point is the universal attractor of the dynamics for finite surface tension.

In Fig. 9 the projection for decreasing values of $\widetilde{B}$ is plot- 


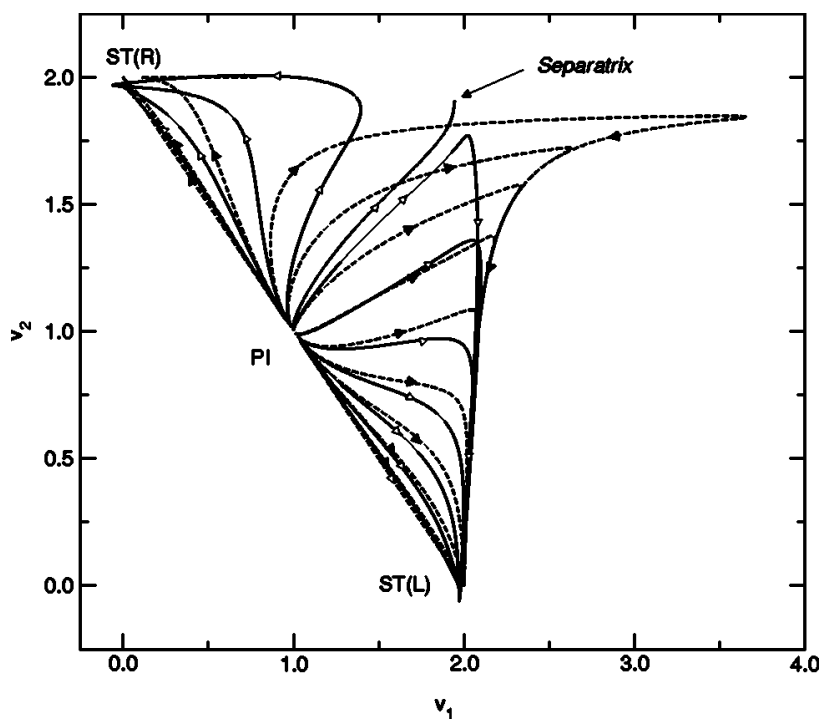

FIG. 10. Plot of the evolution of initial conditions of the form (4) with $\lambda=1 / 2, \epsilon=0.1$, and $\zeta_{s}^{2}(0)=20 \exp ($ in $\pi / 12)$ and $n=0$, $\pm 1, \ldots, \pm 6$ in the $\left(v_{1}, v_{2}\right)$ or tip speed space. The solid line corresponds to $\widetilde{B}=0.01$ and the dashed line to $\widetilde{B}=0$. The computed trajectory that most nearly separates the two basins of attraction is also plotted. Note that the long time behavior of the third and fourth $\widetilde{B}=0.01$ curves (counting from the upper left trajectory in clockwise direction) is dramatically different from the corresponding $\widetilde{B}=0$ solutions.

ted, using the initial condition $\zeta_{s}^{2}(0)=20 \exp (i \pi / 6)$. As $\widetilde{B}$ is decreased the projected trajectory gets closer to the $B=0$ trajectory, but as it approaches the point when the daughter singularity impinges the unit circle (this point is signaled by a cross) the projection departs from the $B=0$ trajectory and approaches the Saffman-Taylor fixed point, consistent with asymptotic theory.

\section{B. Solutions with $\epsilon \neq 0$}

The continuum of fixed points present for $\epsilon=0$ is absent for $\epsilon \neq 0$, but in this case finite-time singularities in the form of zeros of $z_{\zeta}$ impinging on the unit disk do appear for some initial conditions. Therefore, we can expect that the effect of finite surface tension will be somewhat different than for $\epsilon$ $=0$. First, the presence of surface tension should eliminate finite-time singularities, and secondly, finite $B$ could modify the basin of attraction for the two attractors of the $B=0$ dynamical system, namely the side Saffman-Taylor finger and the center Saffman-Taylor finger.

To explore this, we have performed computations with $\lambda=1 / 2$ and $\epsilon=0.1$ with initial conditions $\zeta_{s}^{2}(0)$ $=20 \exp ($ in $\pi / 12)$ and $n=0, \pm 1, \ldots, \pm 6$. Initially we set $\widetilde{B}=0.01$ and use a value of the noise filter level equal to $10^{-13}$, which suffices due to the relatively large value of $\widetilde{B}$. The easiest way to compare both dynamics, finite $B$ and $B=0$, is to plot their trajectories in velocity space. Thus, in Fig. 10 the tip velocities $\left(v_{1}, v_{2}\right)$ of the $\widetilde{B}=0.01$ computation are plotted together with the tip velocities for $B=0$. For arbitrary $\epsilon$ and $\lambda$ the tip velocities of the $B=0$ solution read

$$
\begin{aligned}
& v_{1}=\frac{1+\mathrm{i}\left(\zeta_{s}^{2}-\bar{\zeta}_{s}^{2}\right)+\zeta_{s}^{2} \bar{\zeta}_{s}^{2}}{\zeta_{s}^{2} \bar{\zeta}_{s}^{2}-\epsilon\left(\zeta_{s}^{2}+\bar{\zeta}_{s}^{2}\right)+\mathrm{i} \lambda\left(\zeta_{s}^{2}-\bar{\zeta}_{s}^{2}\right)-(1-2 \lambda)}, \\
& v_{2}=\frac{1-\mathrm{i}\left(\zeta_{s}^{2}-\bar{\zeta}_{s}^{2}\right)+\zeta_{s}^{2} \bar{\zeta}_{s}^{2}}{\zeta_{s}^{2} \bar{\zeta}_{s}^{2}+\epsilon\left(\zeta_{s}^{2}+\bar{\zeta}_{s}^{2}\right)-\mathrm{i} \lambda\left(\zeta_{s}^{2}-\bar{\zeta}_{s}^{2}\right)-(1-2 \lambda)} .
\end{aligned}
$$
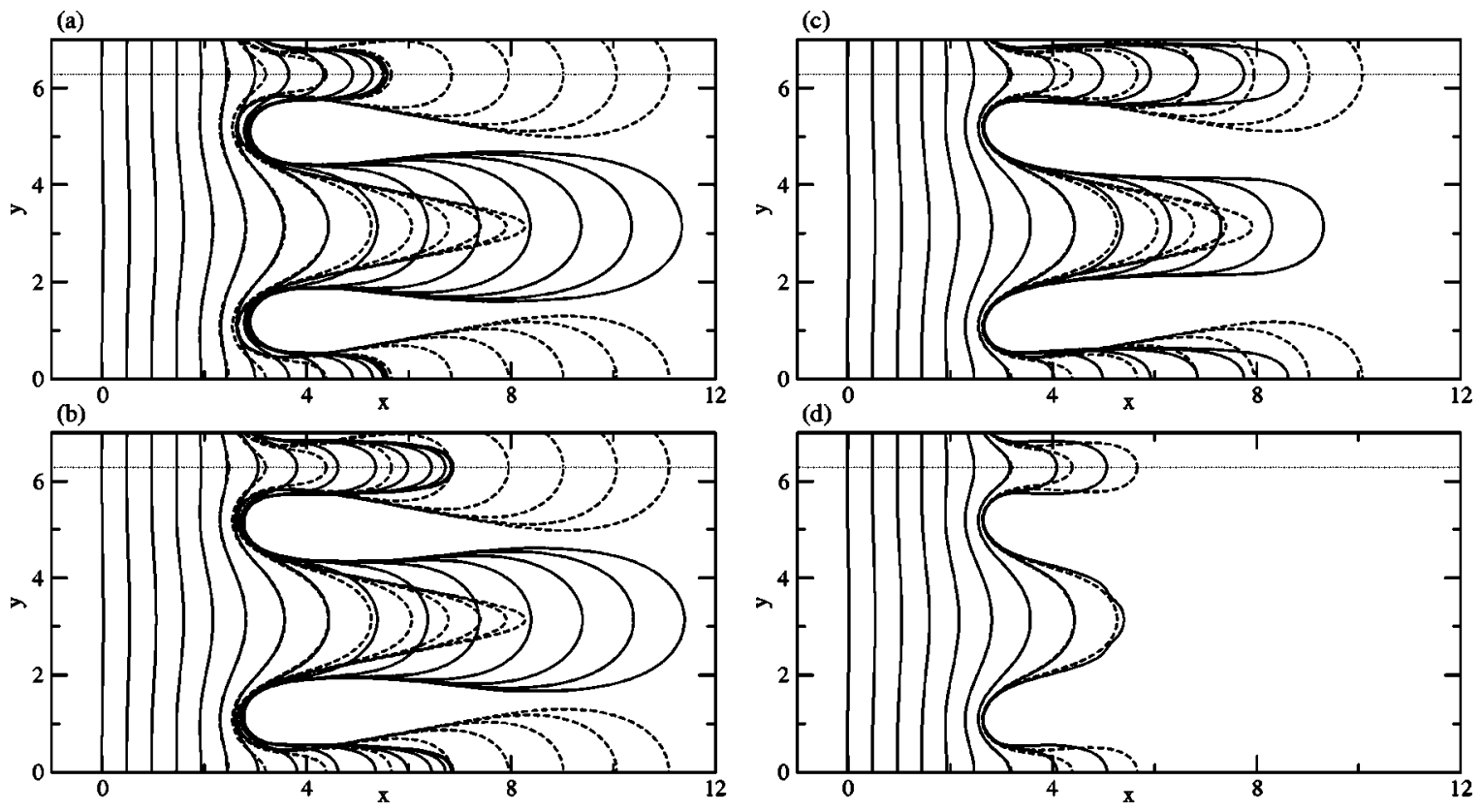

FIG. 11. Evolution of an initial condition of the form (4) with $\lambda=1 / 2, \epsilon=0.1$, and $\zeta_{s}^{2}(0)=20 \exp (-i \pi / 6)$. The solid lines correspond to surface tension $\widetilde{B}$ values (a) 0.01 , (b) 0.005 , (c) 0.001 , and (d) 0.0005 . The dashed lines correspond to the zero-surface-tension evolution. The time difference between different curves is 0.5 . The physical channel in the $y$ direction extends from the origin to the dotted line. 


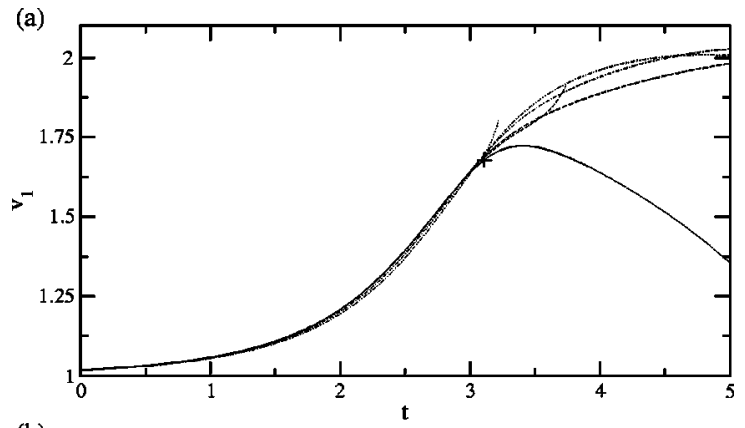

(b)

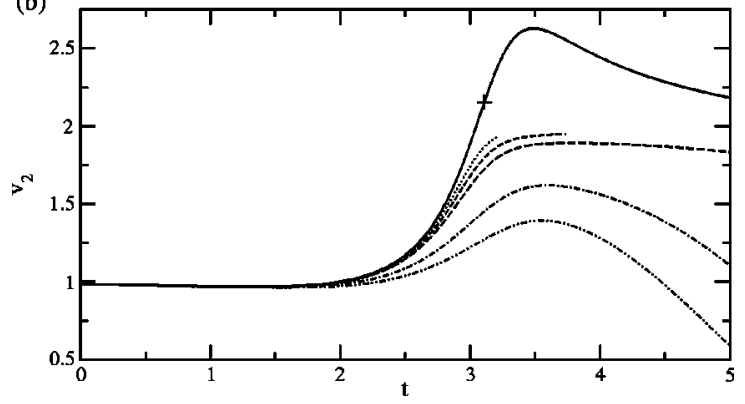

FIG. 12. Computed tip velocities for the initial condition of Fig. 11: (a) corresponds to the central finger and (b) to the lateral finger. The daughter singularity impact time $\tilde{t}_{d}$ is indicated by the + symbol. The value of $\widetilde{B}$ is: 0 (solid line), 0.0002 (dotted line), 0.0005 (dashed line), 0.001 (long dashed line), 0.005 (dot-dashed line), and 0.01 (dot-dot-dashed line). The deviations observed at late times for $\widetilde{B}=0.0002$ and $\widetilde{B}=0.0005$ in (b) are due to numerical noise.

From the plot one can see that most $\widetilde{B}=0.01$ velocity trajectories follow (at least qualitatively) their $B=0$ counterparts, in the sense that they end up in the same fixed point. However, the second, third, and fourth trajectories (counting from the upper left trajectory in clockwise direction) differ significantly from their $B=0$ counterparts. The second $\widetilde{B}=0.01$ trajectory moves apart from the $B=0$ solution simply because the latter develops a finite-time singularity, which is regularized by the introduction of finite surface tension. However, the third and fourth trajectories exhibit a quite surprising behavior: the computed interface with $\widetilde{B}=0.01$ ends up in a different fixed point than the exact $B=0$ solution, despite the fact that the $B=0$ solution is smooth for all time and has the asymptotic width that would be selected by vanishing surface tension.

In order to get further insight into this behavior we have computed the evolution for decreasing values of $\widetilde{B}$ using the specific initial pole position $\zeta_{s}^{2}(0)=20 \exp (-i \pi / 6)$, with $\lambda$ $=1 / 2$ and $\epsilon=0.1$. Quadruple precision has been used when it has been necessary. Figure 11 shows its evolution for four values of the surface tension parameter, together with the $B$ $=0$ solution. The differences between the two interfaces for long times are readily apparent. When $B=0$ the finger in the central position stops growing and the side finger wins the competition, whereas for $B>0$ we encounter the opposite situation-namely, the central finger surpasses the side finger and wins the competition. For the smaller values of $B$ the finger on the sides has not quite stopped growing when the computation is stopped, although its tip speed shows a

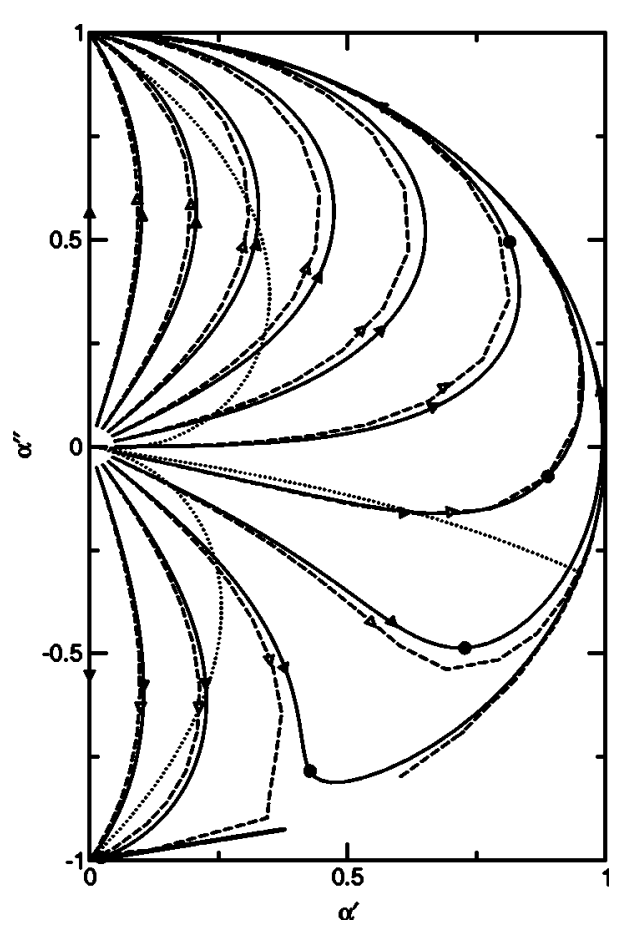

FIG. 13. Comparison between the $\widetilde{B}=0$ trajectories and the projected evolutions with $\widetilde{B}=0.01$, for the initial conditions of Fig. 11 . The solid line corresponds to $\widetilde{B}=0$ and the dashed line to the projection of the $\widetilde{B}=0.01$ evolutions. The daughter singularity impacts are indicated by a circle. Note that the fourth $B>0$ trajectory (as measured counterclockwise from the bottom) reverses direction and heads toward the fixed point $(0,-1)$.

marked decrease over that for $B=0$ and is less than that of the central finger. The side finger tip speed is also decreasing at the final stage of the computation. The tip speed trend in the limit $B \rightarrow 0$ is further illustrated in Fig. (12). This figure shows the tip speed versus time of each finger for a sequence of decreasing $B$. The plot suggests that upon impact of the daughter singularity the side finger velocity levels off and eventually decreases, whereas the velocity of the center finger is nearly unaffected and continues to increase. The trend is indicative of the center finger "winning" the competition in the $B>0$ dynamics, while the opposite occurs for $B=0$. Finally, it is noted that the influence of surface tension is first felt by the smaller finger, which is the recipient of the daughter singularity impact. Afterwards the leading finger begins to widen, in a manner consistent with the conjecture in Sec. III. Further remarks on this point are made in Sec. V.

The projection method described in the previous section has been also applied to this case, and the results are displayed in Fig. 13 in the particular case $\widetilde{B}=0.01$. It can be seen that for most trajectories the projection stays close to the $B=0$ curves, even for long times. The daughter singularity impact still leads to $O(1)$ differences between the $B=0$ and $B>0$ solutions, although the impact does not produce changes in the outcome of finger competition. However, as expected some of the trajectories (namely, the third and fourth as measured clockwise from the bottom) do indicate significant qualitative differences in the long time evolution. 


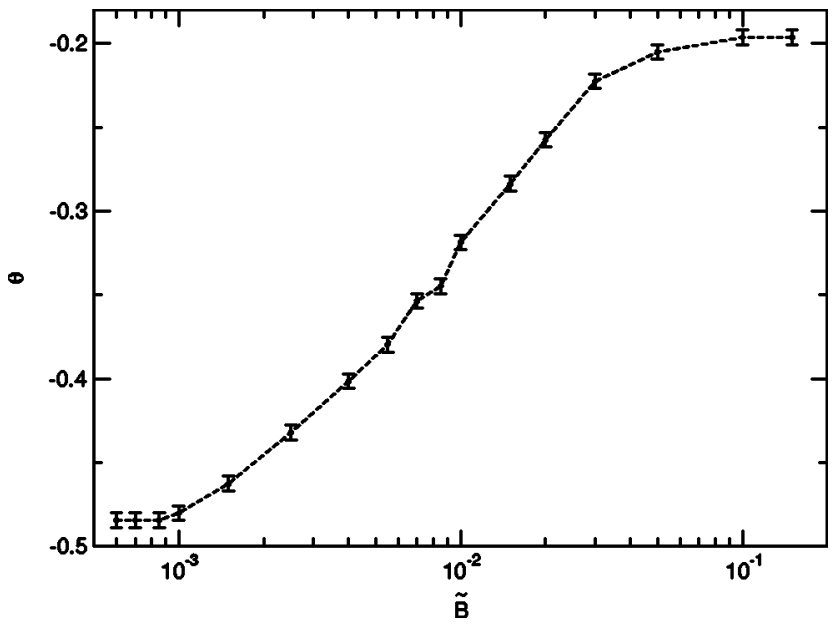

FIG. 14. Plot of $\theta_{\text {sep }}$ versus $\widetilde{B}$ for initial conditions of the form (4) with $\lambda=1 / 2, \epsilon=0.1$, and $\zeta_{s}^{2}(0)=20 \exp (i \theta)$.

The plot provides a simple depiction of the topological inequivalence of the $B>0$ and $B=0$ dynamics [41]

It has been shown that the introduction of a finite $B$ has not changed the attractors of the problem, but it has changed their basins of attraction. Interestingly, in the $B=0$ case there does not exist a single separatrix trajectory between the two Saffman-Taylor attractors, but rather a finite region, corresponding to the set of trajectories ending in cusps, that acts as an effective separatrix. Since for finite surface tension there are no cusps, it can be assumed that there is a single trajectory that separates the two basins of attraction. Obviously, this trajectory will depend on the value of the surface tension parameter. More precisely, the initial condition $\zeta_{s}^{2}(0)$ corresponding to the separatrix trajectory will be a function of the surface tension $B$. To quantitatively characterize this set of initial conditions we have studied the dependence of the separatrix trajectory in a neighborhood of the planar interface fixed point as a function of $\widetilde{B}$, using initial conditions of the form $\zeta_{S}^{2}(0)=20 \exp (i \theta)$. For a given initial condition $\zeta_{s}(0)$ introduce the parameter $\theta_{\text {sep }}(\widetilde{B})$, defined as the unique value for which the evolution is attracted toward the fixed point $\mathrm{ST}(\mathrm{L})$ when $\theta>\theta_{\text {sep }}$ and to the fixed point $\mathrm{ST}(\mathrm{R})$ when $\theta<\theta_{\text {sep }}$.

Figure 14 shows the plot of $\theta_{\text {sep }}$ versus $\widetilde{B}$, and it is observed that as $\widetilde{B}$ decreases, $\theta_{\text {sep }}$ saturates to a fixed value, namely $\theta_{\text {sep }}(\widetilde{B} \rightarrow 0)=-0.4843 \pm 0.0009$. It is interesting to compare this value to the position of the separatrix region for $B=0$, which is located between $\theta_{+}^{B=0}=-0.95758$ and $\theta_{-}^{B=0}=-1.04796$. The separatrix for finite $\widetilde{B}$ lays outside and far away from the separatrix region for $B=0$, even for vanishing surface tension. Our evidence, therefore, suggests that any $B=0$ trajectory located between the trajectories defined by $\theta_{\text {sep }}(\widetilde{B} \rightarrow 0)$ and $\theta_{+}^{B=0}$ will not describe, even qualitatively, the regularized dynamics in the limit $\widetilde{B} \rightarrow 0$, since the finger that will "win" the competition under the $B=0$ dynamics will "lose" under the $B \rightarrow 0$ dynamics. Thus, there exists a positive measure set of initial conditions of the form (4) such that the evolution with $B \rightarrow 0$ cannot be qualitatively described by its evolution under $B=0$ dynamics. This is a dramatic consequence of the singular nature of surface tension on the dynamics of finger competition that is not related to steady state selection, but confirms the ideas of the proposed dynamical solvability scenario in Ref. [22].

\section{SUMMARY AND CONCLUDING REMARKS}

The asymptotic theory developed in Refs. $[13,20]$ predicts the existence of regions of the complex plane where the zerosurface-tension solution and the finite surface tension solution differ by $O(1)$. These regions are the daughter singularity clusters, and their influence is felt in the physical interface when they are close to the unit circle. Daughter singularities move towards the unit circle, and when their motion is not impeded by other singularities they reach the unit circle in $O(1)$ time. When the distance between the daughter singularity and the unit circle is $O\left(B^{1 / 3}\right)$ the interface can display $O(1)$ discrepancies with respect the interface of the $B=0$ solutions.

Since the precise effect of the daughter singularity cannot be established by the asymptotic theory it is necessary to use numerical computation in order to establish the effects of daughter singularity on the dynamics of the interface. We have focused our efforts on uncovering the role of surface tension in the dynamics of two-finger configurations, and two different types of two-finger zero-surface-tension solutions have been studied. The first type $(\epsilon=0)$ does not exhibit finger competition when $B=0$ but rather contains asymptotic configurations consisting of two unequal steady fingers advancing with the same speed. Numerical computations with small surface tension show that the introduction of a small $B$ triggers the competition process which was absent for $B=0$ by restoring the saddle-point (hyperbolic) structure of the appropriate multifinger fixed point. The second type $(\epsilon \neq 0)$ of two-finger solution we have studied exhibits finger competition for $B=0$, but the numerical computation with small $B$ has shown that the long time configuration of the computed interface is qualitatively different from the $B=0$ solution for a broad set of initial conditions, in the sense that the finger that "wins" the competition is not the same with and without surface tension. Thus, the presence of surface tension seemingly can change the outcome of finger competition even in configurations that are well behaved and smooth for all time and whose asymptotic width is fully compatible with the predictions of selection theory for vanishing surface tension. This unexpected result shows that surface tension plays also an essential role in multifinger dynamics through a drastic reconfiguration of the phase-space flow structure.

Our calculations support the conjecture that impact on either the shorter or larger finger retards the velocity of that finger, and is accompanied by the widening of the larger finger. As a consequence, in general the outcome of finger competition is independent of the particular finger on which the impact first occurs, and the finger which is leading at the time of the daughter singularity impact "wins" the competition. This recipe fails only for interfacial configurations with very similar fingers, when not only the position of the finger 
(which finger is leading) but also the tip velocities (a trailing finger can have for a certain time a larger velocity than the leading one) at the impact time may play a role.

The main conclusion of the present work is that surface tension is essential to describe multifinger dynamics and finger competition, even when the corresponding zero-surfacetension evolution is well behaved and compatible with selection theory. That is, we have detected singular effects of surface tension on the dynamics of finger competition that are not directly related to steady state selection. These can be properly interpreted in the context of an extended dynamical selection scenario as described in Ref. [22], where the reconfiguration of phase-space flow by surface tension can be traced back to the restoring of hyperbolicity of multifinger fixed points.

\section{ACKNOWLEDGMENTS}

We acknowledge financial suport from the Dirección General de Enseñanza Superior (Spain), Project No. BXX20000638-C02-02 (J.C. and E.P.) and National Science Foundation Grant Nos. DMS-9704746 and DMS-0104350 (M.S.). E.P. also acknowledges financial support from the Departament d'Universitats, Recerca i Societat de la Informació (Generalitat de Catalunya), and the kind hospitality of the Mathematics Department of the New Jersey Institute of Technology.
[1] P.G. Saffman and G.I. Taylor, Proc. R. Soc. London, Ser. A 245, 312 (1958).

[2] D. Bensimon, L. Kadanoff, S. Liang, B.I. Shraiman, and C. Tang, Rev. Mod. Phys. 58, 977 (1986).

[3] K.V. McCloud and J.V. Maher, Phys. Rep. 260, 139 (1995).

[4] S. Tanveer, J. Fluid Mech. 409, 273 (2000).

[5] J. Casademunt and F.X. Magdaleno, Phys. Rep. 337, 1 (2000).

[6] J.S. Langer Chance and Matter, edited by J. Souletie, J. Vannimenus, and R. Stora, Les Houches (North-Holland, Amsterdam, 1987).

[7] P. Pelcé, Dynamics of Curved Fronts, Perspectives in Physics (Academic Press, New York, 1988).

[8] D.A. Kessler, J. Koplik, and H. Levine, Adv. Phys. 37, 255 (1988).

[9] S.D. Howison, J. Fluid Mech. 167, 439 (1986).

[10] S.P. Dawson and M. Mineev-Weinstein, Physica D 73, 373 (1994).

[11] V.M. Entov, P.I. Etingof, and D.Ya. Kleinbock, Eur. J. Appl. Math. 6, 399 (1995).

[12] F.X. Magdaleno, A. Rocco, and J. Casademunt, Phys. Rev. E 62, R5887 (2000).

[13] S. Tanveer, Philos. Trans. R. Soc. London, Ser. A 343, 155 (1993).

[14] S.D. Howison, J.R. Ockendon, and A.A. Lacey, Q. J. Mech. Appl. Math. 38, 343 (1985).

[15] B.I. Shraiman, Phys. Rev. Lett. 56, 2028 (1986).

[16] D.C. Hong and J.S. Langer, Phys. Rev. Lett. 56, 2032 (1986).

[17] R. Combescot, T. Dombre, V. Hakim, Y. Pomeau, and A. Pumir, Phys. Rev. Lett. 56, 2036 (1986).

[18] S. Tanveer, Phys. Fluids 30, 2318 (1987).

[19] M. Siegel and S. Tanveer, Phys. Rev. Lett. 76, 419 (1996).

[20] M. Siegel, S. Tanveer, and W.S. Dai, J. Fluid Mech. 323, 201 (1996)

[21] F.X. Magdaleno and J. Casademunt, Phys. Rev. E 57, R3707 (1998).

[22] E. Pauné, F.X. Magdaleno, and J. Casademunt, Phys. Rev. E 65, 056213 (2002).

[23] E. Alvarez-Lacalle, J. Casademunt, and J. Ortín, Phys. Rev. E 64, 016302 (2001).
[24] G. Tryggvason and H. Aref, J. Fluid Mech. 136, 1 (1983).

[25] G. Tryggvason and H. Aref, J. Fluid Mech. 154, 287 (1985).

[26] A.J. DeGregoria and L.W. Schwartz, Phys. Fluids 28, 2313 (1985).

[27] A.J. DeGregoria and L.W. Schwartz, J. Fluid Mech. 164, 383 (1986).

[28] T. Hou, J. Lowengrub, and M. Shelley, J. Comput. Phys. 114, 312 (1994).

[29] J. Casademunt and D. Jasnow, Phys. Rev. Lett. 67, 3677 (1991).

[30] J. Casademunt and D. Jasnow, Physica D 79, 387 (1994).

[31] W. Dai and M.J. Shelley, Phys. Fluids 5, 2131 (1994).

[32] H.D. Ceniceros, T.Y. Hou, and H. Si, Phys. Fluids 11, 2471 (1999).

[33] H.D. Ceniceros and T.Y. Hou, J. Fluid Mech. 409, 251 (2000).

[34] G. Baker, M. Siegel, and S. Tanveer, J. Comput. Phys. 120, 348 (1995).

[35] F.X. Magdaleno and J. Casademunt, Phys. Rev. E 60, R5013 (1999).

[36] R. Krasny, J. Fluid Mech. 167, 65 (1986).

[37] For interfaces symmetric with respect to the central axis of the channel our formulation also describes periodic boundary conditions, that is, an infinite array of fingers.

[38] This region can move differently from the $B=0$ (zero) once the second inner region discussed below has impacted the unit disk. We do not consider this possibility here.

[39] Although the daughter singularity is said to impact the unit disk when $\left|\zeta_{d}(t)\right|=1$, the singularities comprising the cluster do not actually impinge upon the unit disk. However, at $t_{d}$ the singularities are close enough to influence the interface shape, in the sense that $\left|z_{\zeta}\left(\zeta_{d}, t_{d}\right)-z_{\zeta}^{0}\left(\zeta_{d}, t_{d}\right)\right|=O(1)$.

[40] The steady state with $\lambda>1 / 2$ is unstable to tip splitting modes, although specifying the initial value problem in the extended complex plane precludes the presence of noise needed to activate the instability.

[41] It is noted that the $B>0$ interfacial profile and the projection profile do differ significantly in small scale features. This is another indication of the difficulties in using zero-surfacetension solutions to describe, even qualitatively, the finite surface tension dynamics. 